\title{
El pronombre en el lenguaje formal del niño
}

\author{
GRACE SHUM \\ Universidad Complutense de Madrid \\ Resumen
}

\begin{abstract}
El uso de la categoría lingüistica del pronombre en el lenguaje verbal del niño es muy temprano, algunas subcategorias de éste aparecen prácticamente desde el primer momento de la aparición de las primeras palabras. No abstante, el adecuado uso anafórico depende del grado de complejidad de cada una de las formas que posee este elemento morfológico. Diversos estudios señalan que en los niños de edad escolar todavía no se encuentra consolidado en alguno de los aspectos esta forma lingüística.

Por tanto, es importante intentar identificar las pautas de adquisición de los pronombres, debido al importante papel que desempeñan en el ámbito lingüístico.

El presente trabajo consiste en el estudio de los pronombres que aparecen en la producción verbal de 180 escolares de $2 .^{\circ}$ curso de EGB, pertenecientes a colegios públicos de las distintas zonas de Madrid. La recogida de datos se realizó a través de una prueba de expresión libre. Se analizaron la producción total de pronombre, y asimismo, se analizaron los errores que aparecen en la narración infantil.

De este modo se pretende precisar aquellos aspectos que suponen mayor dificultad de uso.

Tras el estudio, encontramos que el pronombre, lingüísticamente bablando, constituye uno de los elementos en los que los escolares cometen mayor número de errores sintácticos.
\end{abstract}

Palabras clave: Adquisición del lenguaje, Pronombre.

\section{Pronouns in children's formal languaje}

\section{Abstract}

Pronouns are a linguistic morphological category used in children's verbal languaje from a very early age. Some pronoun subcategories appear practically from the time that the child's first words are uttered. However, its adequate anaphoric use depends on the degree of complexity of each form of this morphological element. Several studies indicate that some aspects of this linguistic form are not fully consolidated in school aged cbildren. Therefore, due to the important vole they play on language, it is necessary to try and establish some guidelines on pronoun adquisition.

The present work studied pronouns appearing in the verbal production of 180 scbool children attending 2 nd grade (7-8 years old) at different state schools in Madrid. A free expression task was used for data collection. The total production of pronouns was analyzed together with errors appearing in the children's narrative. In this way, the study attempted to identify which pronoun subcategories are more difficult to use.

The results showed that, from a linguistic viewpoint, pronouns are one of the elements in which school children make the greatest number of syntactical errors.

Keywords: Language acquisition, Pronoun.

Agradecimientos: El artículo no había sido posible sin la colaboración de Angeles Conde, Carmen Díaz y Francesco Martínez.

Correspondencia con autor-es: Grace Shum. Universidad Complutense de Madrid. Facultad de Psicología. Departamento de Psicología Evolutiva y de la Educación. Campus de Somosaguas. Madrid.

Original recibido: Diciembre 1990. Revisión recibida: Noviembre 1991. Aceptado: Noviembre 1991. 


\section{8}

\section{INTRODUCCION}

El pronombre es una categoría que aparece en la producción lingüística infantil desde una edad muy temprana, prácticamente desde el momento de la aparición de las primeras palabras (Shum; 1986), aunque su adecuada utilización es mucho más tardía. El momento de comienzo de su uso y su correcta producción está determinado por el grado de complejidad de cada una de las formas que toma esta categoría morfológica (tema que ya ha sido tratado por autores como Clark y Sengul; 1978, Charney; 1980, Chiat; 1981). Así, los pronombres demostrativos singulares que indican proximidad y los personales de primera y segunda persona del singular aparecen en torno a los veinte meses, mientras que el uso de los indefinidos es muy posterior (Shum y otros; 1990). Esto es, al principio el niño sólo es capaz de utilizar el pronombre en su función deíctica, ya que si consideramos su función anafórica, encontramos que su producción es posterior (Clark; 1978).

Lingüísticamente, el pronombre es una categoría morfológica que sirve para sustituir la función gramatical que desempeñan otros elementos lingüísticos en la construcción de la oración o el discurso. Como tal categoría carece de significado léxico. Es decir, el significado de cada uno de los pronombres es totalmente dependiente de la situación comunicativa concreta («y» o es la persona que habla, «tu» es el que escucha, «esto» el objeto que está en relación con la primera persona gramatical o que acaba de ser citado, etc.). Estructuralmente, el correcto uso de esta categoría requiere un nivel de conocimiento lingüístico elevado, ya que la utilización de cada tipo de pronombre exige el conocimiento de complejas reglas gramaticales, adecuandose con su elemento antecedente (por ejemplo, los pronombres personales «yo», «tú» y «él» cumplen la función de sujeto para la primera, segunda y tercera persona del singular, mientras que «me», «la» $\mathrm{y}$ «lo» sirven para el complemento directo, $\mathrm{y}$ «me», «le» para el indirecto,). Esto supone que el uso del pronombre implica la capacidad de hacerlo concordar en número y género con el sustantivo y también la capacidad de saber identificar las distintas funciones gramaticales que corresponden a sus distintos tipos (tabla I y II).

Las pautas de aparición de las subcategorías morfológicas del pronombre están estrechamente relacionadas con el nivel de complejidad lingüística que contiene cada una de las formas en que se presenta. Según otros estudios realizados, del que éste es continuación y desarrollo (Shum y otros; 1989, 1990), lo usual es que primeramente surjan los pronombres demostrativos, desde prácticamente la aparición de las primeras palabras, posteriormente los pronombres personales del singular, luego los del plural, y más adelante los indefinidos, los impersonales, etc. Esto es, aparecen, primeramente, los pronombres con función deíctica, mientras que aquellos que poseen un carácter más lingüístico son de aparición mucho más tardía. De todos modos, el momento de uso de cada una de estas subcategorías morfológicas depende del nivel de desarrollo lingüístico del niño. Además, el correcto uso de cada tipo de pronombre está muy condicionado por las características que cada uno de ellos posee, ya que las distintas funciones lingüísticas exigen la intervención de determinadas categorías del lenguaje, y también exigen diferentes niveles de desarrollo de la capacidad lingüística y cognitiva.

Precisamente, por esta razón, ser capaz de utilizar de modo adecuado los distintos tipos de pronombre es esencial en la producción del lenguaje formal, 
TABLA I

El paradigma de los pronombres demostrativos

\begin{tabular}{|c|c|c|c|}
\hline & Masculino & Femenino & Neutro \\
\hline & Sig. PL & Sig. PL & \\
\hline $\begin{array}{l}1 .^{a} \text { pers. } \\
2 .^{a} \text { pers. } \\
3 .^{a} \text { pers. }\end{array}$ & $\begin{array}{cc}\text { este } & \text { estos } \\
\text { ese } & \text { esos } \\
\text { aquel } & \text { aquellos }\end{array}$ & $\begin{array}{cl}\text { esta } & \text { estas } \\
\text { esa } & \text { estas } \\
\text { aquella } & \text { aquellas }\end{array}$ & $\begin{array}{l}\text { esto } \\
\text { eso } \\
\text { aquellos }\end{array}$ \\
\hline
\end{tabular}

TABLA II

El paradigma de los pronombres personales

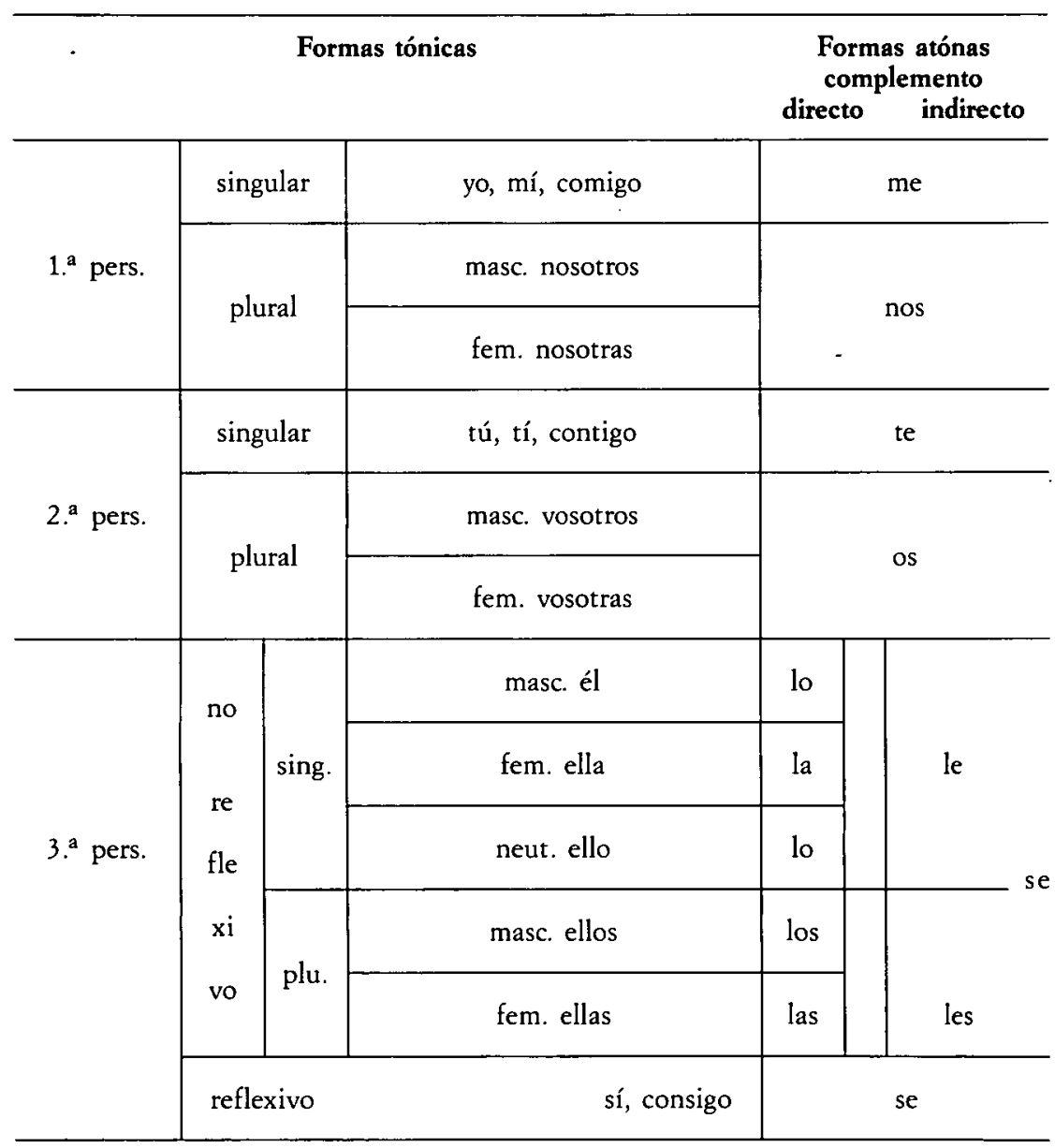


ya que esta categoría morfosintáctica desempeña un papel fundamental, no solo en la elaboración de las distintas clases de oraciones, simples o complejas, sino también como elemento lingüístico clave en la construcción del discurso o del texto. Gracias a la función gramatical de los pronombres se hace posible el establecimiento de distintas formas de conexión oracional, organizando y estructurando lingüísticamente las ideas y expresiones dispersas y discontinuas en oraciones estructuradas de forma adecuada y coherente. Cuando esto no es así, se puede esperar que los errores se presenten, tanto en la expresión como en la comprensión del lenguaje.

Dado el carácter bifuncional de la categoría morfológica del pronombre (deíctico y anafórico), se considera de gran interés la realización de un estudio de las pautas de desarrollo de sus distintas subcategorías en la producción del lenguaje más formal en el marco de estudio de la adquisición del lenguaje. Si se logra identificar los aspectos peculiares de tal proceso de desarrollo, se podrá disponer de mejores elementos explicativos sobre los fenomenos de la adquisición lingüística, lo que ayudará a comprender mejor la relación que existe entre lenguaje y pensamiento.

Por todo lo anteriormente mencionado, se plantea un estudio de las dificultades en la adquisición y desarrollo de la capacidad de uso de los pronombres en la expresión del lenguaje formal. Para ello se intenta identificar los errores morfosintácticos que aparecen en las distintas subcategorías y, asimismo se intenta averiguar el momento en el que se consolida el correcto uso de los distintos tipos de pronombres en la producción lingüística más formalizada de los niños.

\section{METODO Y PROCEDIMIENTO DE ESTUDIO}

Los sujetos del estudio son 180 escolares de segundo curso del primero ciclo de EGB pertenecientes a seis colegios publicos, situados en diversas zonas de Madrid. El objeto de la investigación es el nivel de adquisición y desarrollo del lenguaje formal del niño tras un año de escolarización.

Se realizó una prueba de expresión verbal libre, que consistía en la presentación individual a cada niño de una lámina formada por cuatro viñetas que representaban situaciones fácilmente identificables por él (una casa, un autobús, un aula y un parque), al mismo tiempo que se le daba la consigna de: «cuentame un cuento con estos dibujos», y se tomaba su producción lingüística mediante un aparato grabador de audio. A través de este método se puede unificar el temario de la producción lingüística de los niños estudiados, De este modo, se establece una situación de prueba en la que es posible registrar la producción verbal espontánea de los sujetos, y, dada la inexistencia de diálogo con el experimentador, se exige del niño cierto nivel de formalización lingüística y contenido homogéneo (las mismas viñetas para todos los niños), posibilitando un análisis comparativo entre los sujetos.

Posteriormente, se transcribieron de forma literal todas las historias contadas por los niños incluyendo pausas, muletillas, interrupciones etc. A partir de estas transcripciones fueron extraidos los «textos selectos» por cinco jueces, a fin de evitar valoraciones subjetivas. Estos textos están formados por la expresión verbal de los escolares estudiados, exceptuando aquellos elementos tartamudeos, muletillas, interrupciones, repeticiones, etc.- que, aunque son im- 
portantes para otros tipos de estudios (análisis pragmático del lenguaje por ejemplo) no son significativos para el análisis formal del lenguaje, que, mas bién dificultarían.

Teniendo como base los «textos selectos» se pasó a analizar los errores desde el punto de vista estructual - cometidos por el niño en su expresión del lenguaje verbal, ya que como señalan Crystal y otros autores (1984), en este período de adquisición del lenguaje es más fácil señalar lo que el niño no sabe que lo que conoce. Al mismo tiempo, el análisis de los errores es un campo que genera gran cantidad de hipótesis sobre el propio proceso de adquisición lingüística.

\section{METODO DE ANALISIS}

El análisis consiste en observar los errores gramaticales que se presentan en las distintas subcategorías de pronombres. Para esto se analizan los errores que pueden afectar a la estructura sintáctica. En primer lugar, se determina en qué nivel de la producción lingüística se origina el error, diferenciandose entre el nivel de la oracion y el del discurso. A su vez, dentro del nivel oracional, se distingue el tipo de oración afectada por los errores producidos: oración simple, coordinada y subordinada. A través de este análisis se puede detectar cómo y a qué nivel estructural inciden estos errores morfológicos. El objetivo fundamental al establecer estos dos niveles de análisis estriba en poder distinguir los modos que tienen los niños de cometer errores morfosintácticos con los pronombres, ya que un error cometido en una oración no afecta necesariamente al discurso y viceversa.

Por otra parte, se analizan los diversos tipos de errores que puede presentar cada categoría morfológica, y entre ellas el pronombre. Los errores manifestados pueden ser de los siguientes tipos: omisión, inadecuación, sustitución, adición, de orden y de concordancia. En relación con el pronombre pueden ser definidos como sigue:

Omisión, cuando algún pronombre es omitido ya sea en la oración o en el discurso, siendo necesario en la construcción de la oración o el discurso.

Inadecuación, cuando el pronombre ha sido utilizado deforma equivocada, tanto en la oración como en el discurso, y cuando, además, en lugar de tal equivocación se pueden encontrar varias posibles alternativas para la elaboración de una oración o de un discurso correcto.

Sustitución, cuando un pronombre ha sido sustituido por otro elemento morfológico, bien sea de la misma categoría o de otra categoría distinta.

Adición, cuando el uso del pronombre es incorrecto por ser innecesario.

Orden, cuando el lugar correcto que le corresponde a un pronombre en una oración ha sido alterado.

Concordancia, es el caso en el que el pronombre no concuerda con los demás categorías, en número, género o persona.

Se analizan los errores que aparecen en las subcategorías del pronombre con el fin de identificar las pautas y el momento de adquisición de cada una de ellas. A través de este análisis se comprenden mejor las características y complejidad estructural que poseen estos elementos.

También se realiza un análisis morfosintáctico para precisar los distintos aspectos gramaticales (sujeto, complemento directo, indirecto, etc) en los que el uso del pronombre es erroneo. De este modo se obtiene información sobre el 
grado de dificultad que el niño encuentra en los diferentes tipos de subcategorías del pronombre (demostrativo, personal, posesivo, relativo, indefinido, etc.). Además, se observa el índice de errores que presenta el pronombre en la producción del lenguaje: Se calcula la proporción de errores en el pronombre y en las demás categorías morfologicas (sustantivo, verbo, adjetivo, adverbio, artículos preposición y conjunción) en relación con el total de su producción en el ejercicio lingüístico realizado por los niños. Así, se obtiene una medida del grado de dificultad que encuentran los niños en cada una de las categorías mencionadas.

Por último, se realizan tabulaciones cruzadas y se utiliza el análisis de contingencia para examinar, por una parte, el nivel de relación que existe entre las categorías de la función sintáctica y las morfológicas y, por otra, el grado de dependencia entre los distintos tipos de errores y las diferentes categorías morfológicas. A través de este análisis se puede obtener una idea más clara sobre la relación que mantiene el pronombre con los aspectos sintácticos del lenguaje.

\section{ANALISIS DE LAS PAUTAS DE ERRORES QUE PRESENTA EL PRONOMBRE EN LA PRODUCCION LINGÜISTICA.}

$\mathrm{Al}$ observar la producción lingüística de los niños, en una situación en que estos han de hacer uso de un lenguaje mas formal, distinto al cotidiano que emplean en otros contextos, se comprueba que cometen abundantes errores en el uso del pronombre.

A partir de la tabla de frecuencias de los errores que los niños producen en las diferentes categorías morfológicas, se puede decir que la categoría de pronombre es uno de los elementos en el que los escolares cometen mayor número de incorrecciones estructurales (tan solo menos que el verbo, 9 puntos menos y que el sustantivo, algo más de 1 punto menos). Sin embargo, hay que tener en cuenta, que el verbo y el sustantivo son las categorías más usadas en la construcción de las oraciones, ya que la primera es el elemento gramatical imprescindible en la elaboración de cualquier oración y la segunda es, fundamentalmente, un elemento que contribuye a la semantización, lo que constituye el eje esencial del significado en la oración. Así, por tanto, son categorías morfológicas que siempre presentan mayores frecuencias en cualquier producción lingüística, y lógicamente, suponen también mayor porcentaje de incorreciones sintácticas. Contrariamente, la categoría del pronombre no sólo tiene por característica sustituir al sustantivo sino que, como indica Coseriu (1978), o Porto Dapena (1986), es una categoría transversal aplicable en ciertas unidades lingüísticas que funcionan como sustantivo, adjetivo o adverbio. Esto quiere decir que el uso de esta categoría no sólo tiene lugar después de la aparición del sustantivo (lo que implica una relación coherente y ordenada, en género, número y persona, con los sustantivos expresados), sino que tambíen puede presentarse como adjectivo, (por ejemplo el uso de los pronombres demostrativos) y como adverbio (como, según Marcos M. (1978), los adverbios deícticos: «aquí», «allí», «acá», «allá», etc.) Consecuentemente, el correcto uso de los pronombres en la producción del lenguaje formal exigiría un mayor desarrollo y consolidación lingüística y cognitiva, aunque es utilizado desde prácticamente el primer momento de la aparición del lenguaje en la comunicación contextual.

La comprobación de lo anteriormente dicho la encontramos en que, aunque el número de errores del pronombre es menor que en el verbo y el sustantivo 


\section{3}

en terminos absolutos (figura 1), en relación con la producción total de cada una de las categorías morfológicas el uso del pronombre alcanza un porcentaje de errores cercano al $20 \%$, muy superior al de cualquier otra categoría, ya que en el verbo la proporción de errores sobre el total de verbos producidos es del $12 \%$ y en el sustantivo de tan solo el $6 \%$; estando las demás categorías morfológicas entre el 3 y el $9 \%$ (figura 2).

FIGURA 1

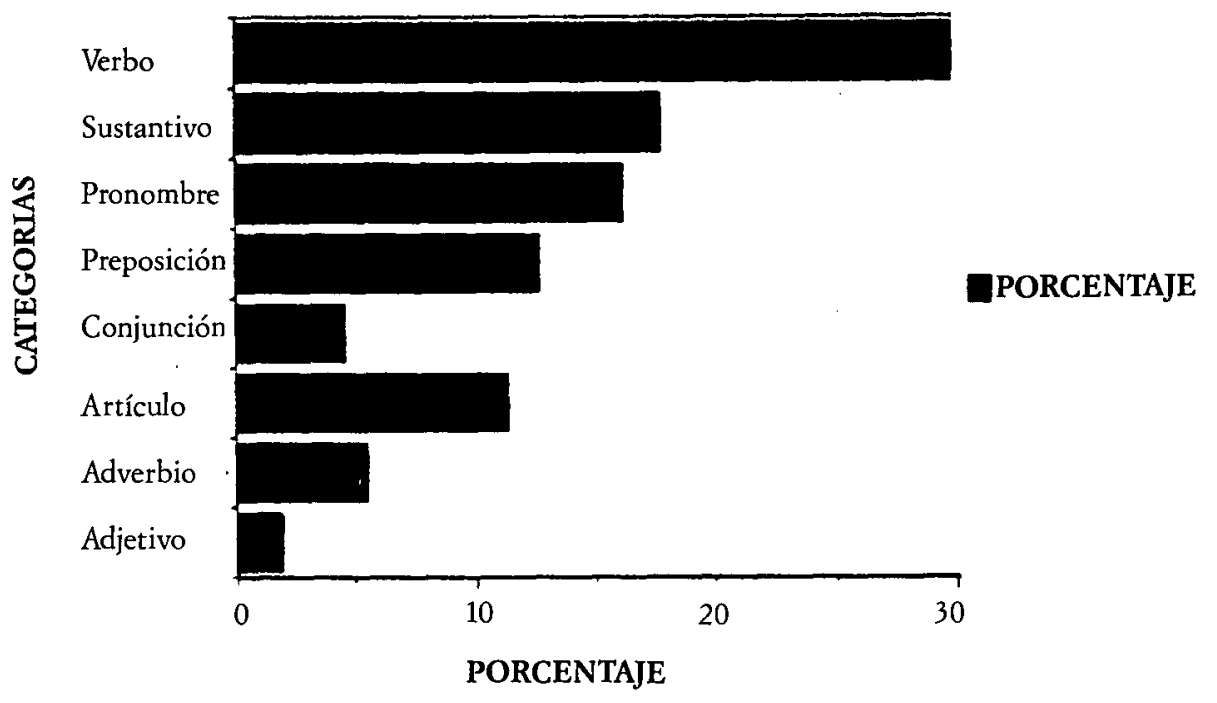

Distribución de errores por categorias morfológicas

Figura 2

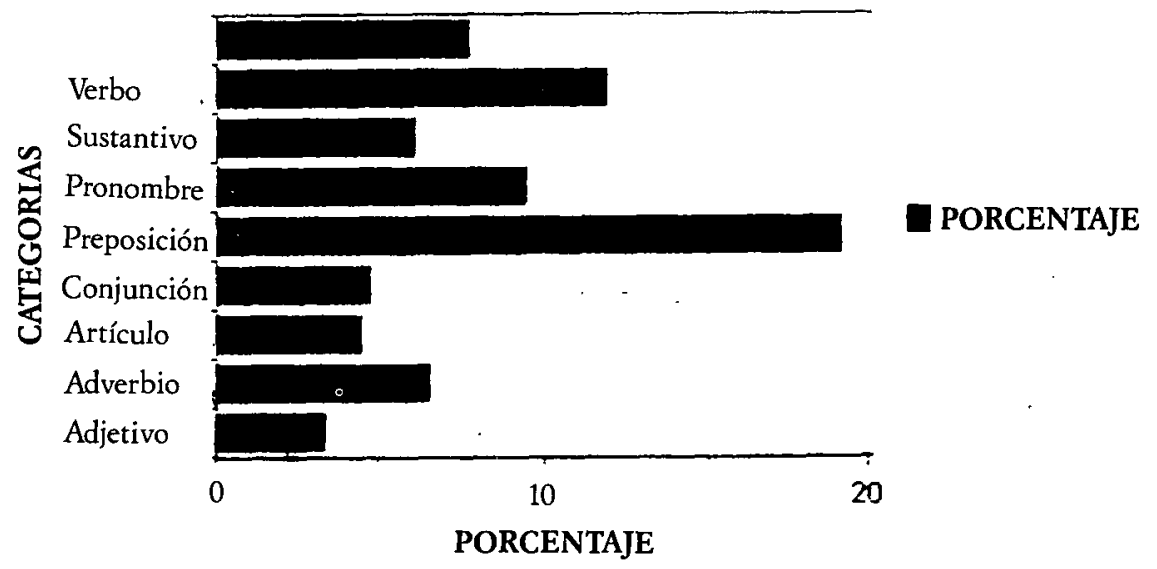

Porcentaje de errores morfológicos sobre la producción lingüistica 


\section{4}

Con respecto a las subcategorías morfológicas del pronombre, se observa que los niños presentan mayor porcentaje de errores en demostrativos (47\%), los pronombres personales se sitúan en segundo lugar (37\%) y, por último, los reflexivos, que suponen un $8 \%$ del total. En cuanto a los demás tipos de pronombres, como los posesivos, indefinidos y relativos, representan una proporción mínima de los errores del pronombre, ya que la producción de tales formas también es muy escasa (figura 3). Esto se debe, a la prueba utilizada que consiste en la construcción de un discurso a través de unas viñetas, por lo que el niño no está obligado a utilizar determinados términos morfológicos. No obstante, también hay que tener en cuenta que la expresión de estos últimos tipos de pronombres, en el uso general del lenguaje, es menos frecuente y, también, por esta misma razon el correcto uso de tales términos lingüísticos es mas tardío.

El hecho de cometer muchos errores en el uso de los pronombres demostrativos, siendo su utilización muy temprana y no detectándose apenas errores de uso en la comunicación conversacional ya en torno a los dos años y medio (Shum y otros; 1990), es prueba suficiente de que en los niños de segundo curso de EGB todavía no se encuentra consolidado el uso del lenguaje formal. Por esta razón, cuando se les exige una expresión en lenguaje más formalizado, presentan errores morfosintácticos en dichos términos, ya que la adecuada producción en la lingüística formal no sólo requiere una comprensión del significado de estos elementos, sino el conocimiento de leyes sintácticas que implican el pleno conocimiento de las relaciones espaciales, de cantidad, número y género.

Del mismo modo, cabe decir que los errores que los niños producen en el uso de los pronombres personales no son sino fruto de la escasa consolidación del lenguaje escolar, ya que dichos errores aparecen, bien en la utilización inadecuada de los verbos reflexivos (por ejemplo: «estoy en la cama, me despierto, $<$ me $>$ cojo la cartera»), bien en el incorrecto uso de la función gramatical de complemento directo e indirecto, que ya ha sido expresada mediante un sustan-

Figura 3

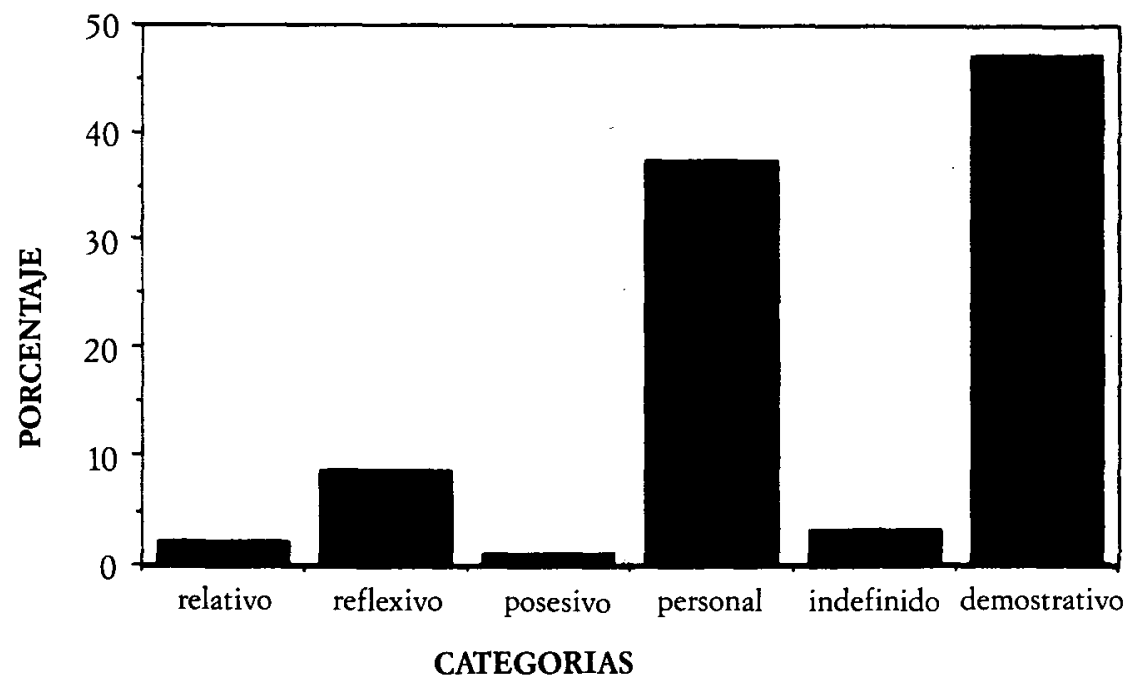

Distribución de errores del pronombre por subacategorias 
tivo. Esto es, el niño construye el complemento directo e indirecto de la oración a través del sustantivo y al mismo tiempo añade el pronombre para tal función (por ejemplo: «<los $>$ lleva al colegio a los niño»). Por último, hay otro tipo de errores de pronombres que pertenecen al habla regional, del tipo del leismo y laismo, siendo aceptado en la expresión del lenguaje coloquial, pero incorrecto en el lenguaje formal.

En el presente estudio se detecta que los escolares emplean fundamentalmente los pronombres de la tercera persona singular y que los errores que producen son en su función de objetos directos o indirectos. Esto es, en linea general, el niño comete errores estructurales, cuando utiliza el pronombre para la construcción del elemento del complemento directo e indirecto. Estos tipos de errores están muy relacionados con el nivel de complejización que aparece en las oraciones, cuanto más complejas son las oraciones, mayor número de errores presentan. Esto no es sino fruto de la escasa consolidación y adquisición del conocimiento sintáctico del lenguaje, por lo que el niño todavía no es capaz de discriminar la función deíctica de la anafórica; de tal modo que el niño confunde el uso del género, número, objeto directo e indirecto, etc. Estos errores se pueden observar muy bien a través de las siguientes oraciones-tipo:

Errores del objeto directo:

$-<$ los $>$ lleva al colegio a los niños.

$-<$ le $>$ espero al autobus.

-se fue con su cartera que se $\langle$ la $\rangle$ dejó en el banco para columpiarse.

-nunca mas $<$ la $>$ volvió a encontrar la cartera.

$-<$ les $>$ fue a buscar $<$ les $>$ y $<$ les $>$ encontró en el parque.

Errores del objeto indirecto:

$-<\mathrm{la}>$ pusieron mesas y silla.

-se fue con su cartera que <se > la dejó en el banco para columpiarse.

- dijo el niño <me $>$ se ha olvidado en el parque la cartera.

- y se $<$ le $>$ dejó una cartera.

Estos errores aparecen generalmente en el uso del pronombre personal, «me», «te», «lo», «le» y «se», en el momento en que desempeña la función del complemento directo e indirecto. Este tipo de errores gramaticales se manifiestan, sobre todo, cuando se utilizan tales morfologías para la elaboración de las oraciones con los verbos : «gustar»y «querer», ya que dichos verbos presentan, de forma muy explícita, el sujeto y el complemento. Además, hay errores que se cometen con el uso de los verbos, esto es, que el niño confunde el verbo no reflexivo con el reflexivo y, en tal caso, el niño suele añadir el pronombre personal en la oración expresada. Estos errores se presentan de los siguientes modos:

-fue el autobus y $<$ se $>$ le llevó al colegio.

- estoy en la cama, me despierto, <me > cojo la cartera.

- cuando paró < se> fue, se metió en él y le llevó al cole.

Mediante el análisis de contingencia se observa que existe una asociación significativa entre las categorías de la función sintáctica y las categorías morfológicas (siendo la prueba de la Ji-cuadrado significativa con un nivel de error del $0.1 \%$ y el coeficiente de contingencia del 0.52 ), lo cual supone que hay una estrecha relación entre los elementos morfológicos y las leyes gramaticales. Entre los errores morfológicos que aparecen en toda la producción lingüística de los niños se observa que al pronombre corresponde un $15 \%$ del total. El mayor número de incorrecciones que se cometen en las funciones de complemento directo e indirecto corresponden al pronombre ( $48 \%$ y $87 \%$ respectivamente), 
mucho más que cualquier otra categoría morfológica (tabla III). Este hecho se explica, precisamente, por la mayor complejidad de elaboración de tales funciones cuando se usa el pronombre, ya que para ello se exige la transformación de la morfología, así el «yo», «tú», «él», etc. son utilizados para la función de sujeto, mientras que el «me», «lo», «la», etc. sirven para el complemento directo, y el «me», «te», «le», etc. para el indirecto. Su uso correcto implica, realmente, unos conocimientos de las leyes gramaticales muy consolidados.

TABLA III

Porcentaje de errores del pronombre y otras categorias según su función sintáctica

\begin{tabular}{lcr}
\hline & Pronombres & Otras \\
\hline C. Circunstancial & 2,2 & 97,8 \\
C. Directo & 48,5 & 51,5 \\
C. Indirecto & 86,7 & 13,3 \\
Nexo & 1,8 & 98,2 \\
Predicado & 0,0 & 100,0 \\
Sujeto & 25,1 & 74,9 \\
\hline
\end{tabular}

A través también del análisis de contingencia, se observa que existe una correlación de dependencia significativa entre los distintos tipos de errores que producen los niños y las distintas categorías morfológicas (ofreciendo el mismo resultado la prueba de la Ji-cuadrado que en el caso anterior). Entre los errores morfológicos producidos, los pronombres ocupan un $13 \%$ frente a las demás categorías (sustantivo, verbo, adverbio, adjetivo, artículo, preposición y conjunción). Por tipos de errores, aquellos en que los pronombres abundan más los de adición (34\%), concordancia (32\%) e inadecuación (21\%), en que el porcentaje que representan es muy superior al que estadísticamente cabría esperar (tabla IV).

TABLA IV

Porcentaje de errores del pronombre y otras categorias según el tipo de error

\begin{tabular}{lcc}
\hline & Pronombres & Otras \\
\hline Adicción & 34,4 & 65,6 \\
Concordancia & 32,9 & 67,1 \\
Inadecuación & 20,5 & 79,5 \\
Omisión & 3,6 & 96,4 \\
Orden & 12,5 & 87,5 \\
Sustitución & 94,0 & 6,0 \\
\hline
\end{tabular}

Estos resultados se confirman mediante un análisis de tipo cualitativo. Así, los mas frecuentes tipos de errores de los pronombres que se presentan en la expresión lingüística de los niños pueden agruparse de la siguiente forma: 
1. Omisión: Este tipo de errores generalmente suele aparecer en los pronombres demostrativos. En un principio, este hecho hace suponer que tales errores no son sino fruto de la poca consolidación de la adquisición del uso de la función anafórica de esta categoría morfológica. Esto es así, porque los demostrativos en el uso de una comunicación conversacional desempeñan, generalmente, la función deíctica. El uso de tal función no precisa el perfecto conocimiento gramatical. No obstante, hay que tener en cuenta que el tipo de observación que hemos realizado se puede prestar a una situación comunicativa de cierta ambigüedad, ya que existe un apoyo en la situación de prueba (las viñetas) que sirve como referente de la construcción del discurso. De este modo, el niño puede tomar la existencia de tal referente de forma implícita, omitiendo, así, el elemento del sujeto de la oración inicial de su discurso. Se puede señalar este hecho a través de los siguientes ejemplos:

- ( ) es una casa.....

- () son unos niños que salen de su casa.

Como se puede observar, el significado de estas oraciones es completo y perfectamente comprensible para el sujeto receptor en una comunicación interpersonal, ya que el contexto comunicativo en el que se elabora el discurso conlleva implícitamente el referente. Sin embargo, desde el punto de vista de un análisis del lenguaje formal, estas expresiones no son correctas, puesto que son incompletas estructuralmente (falta el sujeto de la oración para completar el sentido del mensaje). Dada la forma de la estructuración de las oraciones expresadas, el elemento lingüístico omitido no puede ser otro sino el pronombre demostrativo.

En todo caso, se puede afirmar que el tránsito del uso de la función deíctica (que aparece en el lenguaje conversacional a una edad muy temprana) a la lingüística es un proceso complejo que requiere cierto nivel de madurez en el uso del lenguaje, por lo que los escolares tienen tantas dificultades en este aspecto.

2. Sustitución: Este tipo de errores aparece, generalmente, en el uso de los pronombres personales. Es decir, el niño confunde la función del complemento directo e indirecto, de tal modo que suele sustituir el uso del objeto directo por el del indirecto y viceversa (por ejemplo: $\langle$ les $>$ cogió en autobus para llevar $<$ les $>$ al cole.).

Este tipo de error puede explicarse mas bien desde una perspectiva sociolingüística que por un problema de desarrollo lingüístico, ya que se trata de una forma dialectal de uso de estas formas pronominales conocidas como «leismo», «laismo» y «loismo».

3. Adición: Esta incorreción de uso consiste en la inadecuada utilización de los verbos. Uno de estos tipos de errores puede ser que el niño confunda él verbo que no es reflexivo por el que sí lo es, (por ejemplo: <se > salió al recreo.), lo que supone una inadecuada utilización de los verbos.

Otros errores de este tipo son los que aparecen en el momento en que el niño presenta una redundancia de uso del pronombre personal, tanto en la función de objeto directo como en la de indirecto, ya que dicha función ha sido expresada, con anterioridad, a través del sustantivo (por ejemplo: <los > lleva al colegio a los niños.).

4. Concordancia: Estos errores consisten en que el pronombre no concuerda con el sustantivo, ya sea en número o género, en la elaboración del mismo 
discurso o en la oración (por ejemplo: /Esto/ era una /casa/ que vivían dos niños.). Dado que la mayoría de los errores de este tipo aparecen entre pronombres demostrativos y sustantivos en construcciones con verbo copulativo, y al inicio del discurso, en que se utilizan pronombres neutros con sustantivos femeninos o masculinos, pudiera tratarse de una generalización de la forma de comenzar la historia.

5. Inadecuación: Estos errores estriban en que el niño comete errores en el uso de pronombres cuyo referente no ha sido explicitado previamente en el texto. Tales errores se cometen, generalmente, con los pronombres indefinidos y demostrativos, como se puede observar en los siguientes ejemplos:

$-<$ uno $>$ que vivía en una casa.

- ya supo que era la «otra» la verdadera suya.

- piensa que es «eso».

Este tipo de errores muestra como el niño que conoce el uso deíctico de determinados pronombres, no conoce, sin embargo, sus características gramaticales, es decir, anafóricas, que requieren el haberse referido con anterioridad a ello dentro del discurso

Por último, cabe comentar que los errores de los pronombres que cometen los niños, en lo que se refiere a la función paradigmáica que dicha categoría cumple, son en su práctica totalidad del tipo que afecta a la referencia del discurso, ya que la elaboración de un discurso siempre supone mayor dificultad que la construcción de cualquier tipo de oración, puesto que su adecuada expresión no sólo requiere una correcta producción gramatical, sino que exige el establecimiento de un nexo, uniendo las oraciones anteriores con las siguientes de forma lógica, para obtener una estructura coherente. Esto se puede señalar a través del siguiente ejemplo:

- Esto era una vez una casita (...) que (vivía) dos ositos. $<$ le $>$ mandó la madre al osito pequeñito a (por) un recado.

Como se puede observar, en este pequeño discurso se presentan errores que afectan tanto al aspecto gramatical como al significado del mensaje. Respecto al primer aspecto, en la primera oración el niño comete errores estructurales. Esto es, no sabe establecer un nexo de forma correcta para el uso del pronombre relativo «que». Además, en lo que se refiere al segundo aspecto indicado, el uso de este relativo sin los elementos de nexo («en») y de determinación («la») que lo acompañen tiene un significado semánticamente distinto. Así, paradigmáticamente se necesita el acompañamiento de otros elementos morfológicos para que el uso sea correcto. Esto es, se necesita utilizar los elementos «en la que» para sustituir «en ella»o «en la casa». En la oración siguiente del discurso el niño también comete dos tipos de errores. Paradigmáticamente, no se puede sustituir el sustantivo «osito» por el pronombre «le», ya que «le» es significativamente distinto a «ies» o «los». Además de ser confuso respecto al referente al que informa, el uso de «le» es incorrecto estructuralmente, porque no es posible la utilización del pronombre «le» para la función de objeto directo

De todo lo dicho, cabe concluir que los pronombres juegan un papel importante ya que la construcción del discurso requiere su intervención, pues desempeñan la tarea de sustituir las distintas funciones sintácticas de los sustantivos, adjetivos y adverbios. No obstante, el correcto uso del pronombre necisita el buen conocimiento de todos los elementos morfológicos y las leyes sintácticas 


\section{CONCLUSION}

Del análisis realizado se deduce que la adquisicón de los pronombres en el lenguaje formal del niño es bastante tardía, aunque el uso de estos términos comienza ya desde una edad muy temprana. Este hecho se puede explicar a través de las funciones que esta categoría morfológica desempeña. Aparte de la función lingüística, que poseen todos los pronombres, hay que considerar también la función deíctica que muchas subcategorías de éste desempeñan. Esta función es muy usada en la interacción interpersonal y en contextos comunicativos concretos y simples. Esto es, los interlocutores pueden servirse de tales subcategorías como vehículo de comunicación para la transmisión e intercambio de información. Dada la simplicidad de su uso, que no exige otra cosa, sino una competencia comunicativa elemental que no precisa una capacidad ni un conocimiento lingüístico elevado, se hace posible su adquisición muy precozmente

Según los resultados del análisis realizado, el pronombre presenta un alto porcentaje de errores sintácticos en la expresión y construcción de un discurso que requiere un uso del lenguaje mas formal. Este hecho se puede explicar por las características específicas de esta categoría morfológica y, ademas, por las exigencias que se plantean en el proceso de construcción del discurso, que son mucho más complejas que las de elaboración de una oración aislada, cualquiera sea el tipo de esta.

Esto es, la sola existencia de la competencia sintáctica no sería suficiente para explicar la correcta producción de un discurso o texto; se necesitaría, además, una adecuada adquisicón de conocimientos en sus distintos tipos y niveles, sociales, cognitivos, etc. Ello implica el alcance de un determinado grado de desarrollo madurativo y un favorable desarrollo del pensamiento y del lenguaje, que sólo un aprendizaje formal permite que se realice en las mejores condiciones posibles, puesto que la construcción de un discurso es una labor muy compleja que exige un nivel de razonamiento y de pensamiento mas abstracto. Tan solo así, se hace posible la estructuración de un relato como el que se exige en la prueba realizada, relacionando, anticipando y concluyendo los distintos elementos, acontecimientos y acciones, de forma lógica y coherente. Tal hecho pone a prueba el pensamiento y el lenguaje formal de los niños, abriendo un campo más extenso de investigación, centrada, más que en los aspectos estructurales del lenguaje, en los referidos a la capacidad del niño para elaborar un discurso cumpleto, en el que aparecen en primer plano de interés los elementos que dan cohesión y coherencia a la intención comunicativa del niño, que merecen considerados en otros estudios de forma más específica.

Lo anteriormente dicho nos hace afirmar que, a pesar de que hay muchas formas del pronombre que aparecen en el niño desde una edad muy temprana y que usa de forma adecuada en la comunicación diádica y en contextos concretos, tal capacidad dé uso pertenece exclusivamente a la función déictica. Esto significa que la adquisición de las formas anáforicas del pronombre, las que constituyen sus funciones lingüísticas en sentido estricto, son más tardías. Además, su adecuada adquisición está relacionada, de alguna forma, con el nivel de desarrollo madurativo, cognitivo y lingüístico. Una vez más el uso de ciertas formas lingüísticas se adelanta al verdadero conocimiento (y en ese sentido adquisición) de los mismos

Dado el alto porcentaje de errores que presenta y las características lingüísticas del pronombre, posiblemente, esta categoría lingüística podría servir para 
la evaluación de la capacidad lingüística de los niños y, por tanto, como eje para la elaboración de programas de enseñanza en esta materia. Ello permitiría optimizar la adquisición del lenguaje académico en los primeros cursos de escolarización con el fin de mejorar el rendimiento escolar

\section{Referencias}

ABRIL, G. (1980). Signo: significado y significante. Madrid: Pablo del Río.

Benveniste, (1970). Problemas de lingüística general.Madrid: FCE.

Bruner. J. (1984). Acción, pensamiento y lenguaje, Madrid, Alianza.

Bühler, K. (1968). Teoría del lenguaje. Madrid; FCE.

Clark, E. V. y Sengul, C. J. (1978). Strategies in the acquisition of deixis. Joumal Child Language, $5,457-475$.

CLARK, E. V. (1978). From gesture to word: on the natural history of deixis in language acquisition. En Bruner J. y Gakton, A.: Human growth and development, Clareton Press, Oxford.

Charney, R. (1980). Speech roles and the development of personal pronoms, Joumal Child Language, 7. 509-528.

CHIAT, S. (1981a). Context-specifity and generalization in the acquisition of pronominal distintions, Journal Cbild Language. 8. 75-91.

Chiat, S. (1981b). If were you and were me: the analysis of pronoms in a pronom-resersing child. Joumal Child Language, 9. 359-379.

Coserru, E. (1978). Gramática, semántica universal, Madrid: Gredos.

Crystal, D. y Otros. (1983). Análisis gramatical de los trastornos del lenguaje, Barcelona: Editorial Médica Técnica.

GiLI Gaya. S. (1961). Curso superior de sintaxis español, Barcelona: Vox.

$E_{\text {RVIN }}$ S. M. (1974). Imitación y cambio estructural en el lenguaje de los niños, en Lenneberg y otros (eds): Nuevas direcciones en el estudio del lenguaje, Madrid: Revista de Occidente.

Hernández Alonso, C. (1975). Sintaxis español, Valladolid: Gredos.

JaKoBson, R. (1975). Ensayo de lingüistica general, Barcelona: Sex Barral.

Karmiloff-Smith, A. \& Inhelder, B. (1981). Si quieres avanzar, hazte con una teoría, Infancia $y$ Aprendizaje, 13. 69-88.

Lozano, J. et al. (1982). Análisis del discurso: bacia una semiótica de la interacción textual. Madrid: Catedrá.

LuRIA, A. R. y Yudovich, I. (1980). Lenguaje y desarrollo intelectual en el niño, Madrid: Pablo del Río.

Marcos Marin, F. (1978). Estudio Sobre el pronombre, Madrid: Gredos.

MARSA. F. (1986). Diccionario normativo y guía práctica de la lengua español.Barcelona: Ariel.

Muñoz. C. (1986). Los pronombres. En Siguan, M. (Coord.): Estudios de psicolingüística, Madrid: Piramide.

Napolitano, G. (1986). Genética de la enunciación. En Siguan, M.(coord.): Estudios de psicolingüística. Madrid: Pirámide.

Porto Dapena, J. A. (1986). Los pronombres, Madrid: Edi-6.

Quilis, A. et al. (1976). Lenguaje española, Valladolid: Gredos.

Sнuм. G. (1986). Psicolingüistica aplicada al estudio de adquisición del lenguaje en niños institucionalizados y niños no institucionalizados. Editorial de la Universidad Complutense de Madrid.

Sium, G. (1988). La influencia del contexto de la interacción en la adquisición del lenguaje, Infancia y Aprendizaje, 43. 37-53.

Shum, G. et al. (1989). ¿Cómo se adquieren y usan los términos deícticos en lengua española? Un estudio longitudinal, Infancia y Aprendizaje, 48 45-64.

StubBs, M. et al. (1976). Análisis del discurso, Madrid: Alianza.

$\mathrm{V}_{\mathrm{AN}}$ DıK, T. A. (1980). Texto y contexto: semántica y pragmática del discurso. Madrid: Gredos.

\section{Extended Summary}

Pronouns are a linguistic morphological category which young children use from the time that they are able to communicate with others through verbal expression. Some of its subcategories appear early in the child's linguistic development. Research indicates that personal and demonstrative pronouns are among the first words spoken by the child. This is because pronouns possess specific characteristics of deictic terms. Hence, it is possible to understand the significance of each pronoun within the communicative context. 
The correct use of pronouns as a linguistic category (linguistic term) is not easy, especially in the case of a small child whose language ability is not yet fully acquired or consolidated. The acquisition and development of this morphology from a linguistic term's perspective is necessary not only to achieve a certain level of communicative, cognitive, and linguistic competence, but also to achieve other related aspects such as physical and social knowledge development. Many investigators indicate that most pronoun subcategories (on linguistic terms) can not be aquired by the child until she/he begins school. For this reason, it would be interesting to study how children acquire pronouns as a linguistic term, and which subcategories are most difficult for child language acquisition. This would certainly give us more information on language pattern development, and perhaps it may help us achieve a better understanding of the phenomenon of language acquisition. This information would also be interesting for better educational programme planning.

The present research work studied the use of pronouns as linguistic terms. Subjects were 180 schoolboys attending 2 nd grade (7-8 years old) at different state schools in the Madrid area.

The study examined children's verbal construction after a year of schooling. Children's verbal expressions were recorded using four pictures: house, garden, classroom, and bank. Thus, all the children narrated a story using the same objects. The characteristics of the verbal production task were also the same: a narrative.

First, the productions of linguistic morphologies appearing in the speech of every schoolboy in the study were analyzed. From this analysis, information on the total number of morphological expressions was obtained. In turn, this allowed us to calculate existing differences on the frequency of each kind of linguistic morphology. Then the pronoun category was related to all other morphologies. Moreover, we studied each type of pronoun subcategory appearing in subjects' sentences and discourse, and examined the following questions: 1) Could our schoolboy sample use them correctly? 2) If this was not the case, When did they make grammatical errors? 3) How did errors occur? Did they relate to sentences or discourse? Later, we tried to identify which subcategories the youngest children found difficult to use.

Results show that pronouns occupy a very important place in language construction. Its correct use is not as easy as is often suggested, even though pronouns are used constantly in our daily lives. 